\title{
Effects of MAL-PDT, ingenol mebutate and diclofenac plus hyaluronate gel monitored by high-frequency ultrasound and digital dermoscopy in actinic keratosis - a randomized trial
}

\author{
M. Arisi, ${ }^{1, \star}$ C. Zane, ${ }^{1}$ M. Polonioli, ${ }^{1}$ C. Tomasi, ${ }^{2}$ E. Moggio,,${ }^{1}$ C. Cozzi, ${ }^{1}$ S. Soglia,${ }^{1}$ S. Caravello, ${ }^{1}$ \\ I. Calzavara-Pinton, ${ }^{1}$ M. Venturini, ${ }^{1}$ M.T. Rossi, ${ }^{1}$ P.G. Calzavara-Pinton ${ }^{1}$ \\ ${ }^{1}$ Department of Dermatology, ASST Spedali Civili di Brescia, University of Brescia, Brescia, Italy \\ ${ }^{2}$ Department of Experimental and Applied Medicine, Section of Industrial Hygiene, ASST Spedali Civili di Brescia, University of \\ Brescia, Brescia, Italy \\ *Correspondence: M. Arisi. E-mail: mariachiara.arisi@gmail.com
}

\begin{abstract}
Background The efficacy for actinic keratosis (AK) clearance of field-directed treatments has been investigated in randomized studies against placebo, but the comparison of results is difficult for several methodological reasons.

Objectives The present study aims to compare efficacy of MAL-photodynamic therapy (MAL-PDT), ingenol mebutate gel (IMB) and diclofenac plus hyaluronate gel (DHA) on multiple AKs assessing a new performance index of efficacy, the cumulative AK area and evaluating dermoscopical and high-frequency ultrasound (HFUS) changes.

Methods Patients with $\geq 5$ Olsen II AKs in a $25 \mathrm{~cm}^{2}$ area of the scalp and face were enrolled and randomized to one of the treatment choices. Number of AKs and cumulative area were assessed before and after treatment. Dermoscopy and HFUS were performed on a single AK and surrounding photo-damaged skin in the treatment area.

Results Cumulative AKs area reduced significantly more with PDT compared to other treatment options and with IMB in comparison to DHA. PDT was also the only treatment option that increased at a significant level the dermal density in both target $A K$ and the surrounding skin and decreased significantly the SLEB thickness in the perilesional skin at HFUS.

Conclusions MAL-PDT is more effective than IMB and DHA for reducing the cumulative AK area which is calculated digitally from 3D pictures and should be the preferred performance index for the evaluation of the efficacy of treatments for AKs, rolling out clinical and dermoscopy evaluation. MAL-PDT improves all HFUS features of chronic photodamages of the dermis of the skin underlying and surrounding the AK spots.
\end{abstract}

Received: 29 July 2019; Accepted: 21 November 2019

\section{Funding Sources}

None.

\section{Conflict of Interest}

None.

\section{Introduction}

Actinic keratosis (AK) is the most frequent UV-induced skin disease in people of Caucasian ancestry. ${ }^{1}$

At clinical examination, it appears as a scaly erythematous patch of variable size and it is classified in the I-III Olsen's scale according to the thickness. ${ }^{2}$ Non-invasive diagnostic techniques, e.g. dermoscopy, ${ }^{3,4}$ in vivo confocal microscopy ${ }^{4}$ and high-frequency ultrasound (HFUS) echography, ${ }^{5,6}$ may be helpful for the early diagnosis, treatment monitoring and follow-up of AKs and the imaging of selected morphological features of the surrounding photo-damaged skin. ${ }^{4,6}$ Nowadays, there is a general agreement $^{7,8}$ that all AKs must be treated because the risk of progression to squamous cell carcinoma (SCC) is independent by lesion thickness. ${ }^{9}$ Furthermore, in the case of multiple and confluent AKs, the surrounding photo-damaged skin must be treated as well ${ }^{10-14}$ because it harbours the so-called field of cancerization with keratinocytes bearing a heavy UV-related mutational burden leading of a high risk of new lesions and relapses. $^{7,8}$

This therapeutic goal can be reached with field-directed drug treatments including photodynamic therapy (PDT) with methyl aminolevulinate (MAL), imiquimod (IMI), 5-fluorouracil 
(5FU), diclofenac plus hyaluronic acid (DHA) and ingenol mebutate (IMB). ${ }^{13}$ The efficacy for AK clearance of the treatments has been investigated in randomized studies against placebo, but the comparison of results from different studies ${ }^{12,15}$ is difficult for several methodological reasons. First of all, clearance is almost always assessed with the only visual inspection and this allows some degree of subjectivity among assessors. In second place, results are given in some trials as a rate of cleared AKs and in other trials as a rate of patients with $100 \%$ (or other lower percentages) of cleared lesions. In addition, both methods can be strongly influenced by the number of lesions per patient and the different ratio of Olsen AKI and Olsen AKII in the treated fields. In addition, different protocols of treatment (sometimes a treatment cycle is repeated if no or partial response is seen after the first one) and different time of follow-up before assessment of results are other main confounding factors. Therefore, it has been suggested that direct comparative studies are needed for a better understanding of efficacy. ${ }^{14,16,17}$

The present randomized comparative single-blinded noninferiority study aims to compare clinical multiple AKs (mAKs) of the face and scalp and surrounding skin treated with MALPDT, IMB and DHA. In order to minimize the subjectivity of the assessors, all lesions were Olsen II AKs and a new performance index of efficacy, the cumulative AK area, has been suggested. ${ }^{18}$ This index was defined by using a dedicated analysis software on $3 \mathrm{D}$ digital pictures, which is able to assess the actual lesions' extent. Finally, HFUS features of the perilesional skin were investigated in order to improve the knowledge of treatment-driven changes of the photo-damaged skin.

\section{Materials and methods}

\section{Patients}

We enrolled consecutive adult patients (phototypes I-IV) with $\geq 5$ Olsen II non-pigmented AKs in a $25 \mathrm{~cm}^{2}$ area of the scalp, forehead and face who presented at the Dermatology Department of the University of Brescia, from August 2017 to August 2018.

Exclusion criteria were concomitant clinically significant unstable medical conditions, active systemic infectious diseases, immunosuppression, chemical dependency or alcoholism, current participation in another clinical study, Olsen's grade I or III $\mathrm{AK}$ and/or invasive tumours within the target $25 \mathrm{~cm}^{2}$ area, known allergies to any molecule in the study drugs, use of photosensitizing drugs, pregnancy or lactation, any other dermatological disease in the treatment area or in a distance of $3 \mathrm{~cm}$, prior topical treatment for AKs within a period of 6 months and likelihood of poor compliance. The study was approved by the Local Ethics Committee (Protocol Number 2909), and it was conducted in accordance with the Declaration of Helsinki. All patients were given verbal and written information on the nature of the study and they signed an informed consent before enrolment.

\section{Treatment procedure}

Patients were randomized with a $1: 1: 1$ allocation with a computer-generated list using random permuted blocks of six to ensure concealment of allocation to three-field treatments: conventional MAL-PDT with 16\% MAL cream (Metvix®; Galderma SA, Lausanne, Switzerland), 0.0015\% IMB gel (Picato gel ${ }^{\circledR}$, Leo Pharma, Ballerup, DK) and 3\% + 2.5\% DHA gel (Solaraze ${ }^{\circledR}$ gel; Almirall Italia, Milan, Italy). Patients and treating physicians were not blinded to group assignment.

At baseline, a $25 \mathrm{~cm}^{2}$ skin area of the face or scalp with at least 5 Olsen II Aks was selected ${ }^{2}$ and marked with black ink. All treatments were delivered according to the protocol that is approved by the European Medicines Agency (EMA). IMB gel was self-applied by patients to the target $25 \mathrm{~cm}^{2}$ area overnight for three consecutive days. ${ }^{19}$ With DHA gel and MAL-PDT, the $25 \mathrm{~cm}^{2}$ area was selected within a $200 \mathrm{~cm}^{2}$ treatment area. A fingertip unit (approximately $0.5 \mathrm{~g}$ ) of DHA gel was self-applied twice daily for 90 days. ${ }^{20}$ The first session with MAL-PDT was delivered to all patients and a second session was delivered only if needed after 3 months. ${ }^{21}$

Three performance indexes were used: the number of lesions defined after visual inspection and dermoscopical examination (Vidix 4.0; Medici Medical, Modena, Italy) at baseline (T0) and 90 days after the end of treatment (EOT) (T1) by two investigators (MA and MP) who were blinded to treatment assignment; the AKs' cumulative area in the sample ${ }^{18}$ area calculated with the analysis (Vectra Viewer ${ }^{\circledR}$ software; Canfield, Fairfield, NJ, USA) of 3D digital pictures (Vectra $\mathrm{H} 1{ }^{\circledR}$ camera; Canfield, Fairfield, NJ, USA) and the number of patients attaining the clearance of all lesions (complete responders) in the $25 \mathrm{~cm}^{2}$ treated area.

In addition, we selected a representative Olsen grade II AK and a small spot of clinically unaffected skin within the test area for HFUS investigations (Fig. 1). We used a $50 \mathrm{MHz}$ HFUS (DUB® Skin Scanner; Taberna Pro Medicum, Im Dorf, Lüneburg) that has $4 \mathrm{~mm}$ useful depth of signal penetration and $40 \mathrm{~dB}$ gain under standard conditions $\left(20-23^{\circ} \mathrm{C}\right.$ environmental temperature with patient lying for at least $10 \mathrm{~min}$ ). Total, epidermal and dermal thicknesses $(\mu \mathrm{m})$, subepidermal low-echogenic band (SLEB) thickness $(\mu \mathrm{m})$ and lesional and SLEB echogenicity (expressed as percentage of dermal density) were analysed with a specific image-analysis software as main HFUS features of photodamage or hyperkeratosis (Fig. 1). HFUS investigations were repeated in the same skin spots that were identified on the basis of the digital pictures.

Analysis of 3D digital pictures and HFUS images was performed by two physicians (MA and MP), who were blinded to treatment assignment.

\section{Statistical analysis}

Statistical analysis was performed with the SPSS $^{\mathrm{TM}}$ (v25.0; IBM SPSS, Armonk, NY, USA) software program. Based on previous data on effectiveness of drug treatments ${ }^{16}$ for AKs, assuming a 


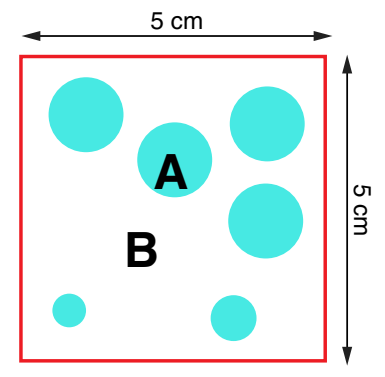

Figure 1 Trial design: we evaluated the AKs number and their cumulative area in a selected $25 \mathrm{~cm}^{2}$ area. In addition, we assessed echographic (total, epidermal and dermal thickness; dermal density) features of a target AK area (A) and echographic (SLEB thickness and density) features of a representative photo-damaged skin area (B). AK, actinic keratosis.

minimal clinical difference of $14 \%$, a standard deviation (DS) of 28 , a power of $91 \%$ and an $\alpha$ value of 0.01 , the sample size required to show a statistical difference was 30 patients for each treatment group.

Categorical variables were summarized using percentages, and continuous variables by calculating medians and range (minimum and maximum values).

Normal distribution of collected data was analysed by the Kolmogorov-Smirnov test.

The medians of continuous variables were compared using the Wilcoxon test.

Changes in clinical and ultrasound features were defined using the non-parametric Kruskal-Wallis test for unpaired data. Only in case of significance, the comparison of each treatment was then defined by Mann-Whitney test.

The same variations were studied with respect to age, using non-parametric correlations with Spearman's Rho, body site, through ANOva parametric test and phototype, with KruskalWallis test.
The identification of any predictors instrumental features of therapeutic response was performed using logistic or nominal regressions.

All results were considered statistically significant at a $P \leq 0.05$ level.

\section{Results}

Ninety patients were enrolled in the study, 30 for each group. Eighty-four patients (93.3\%) completed the study: 26 (28.9\%) were treated with MAL-PDT, $30(33.3 \%)$ with IMB and 28 (31.3\%) with DHA. Six (6.7\%) patients were lost at follow-up for reasons unrelated to treatment.

The following analyses were per-protocol because drop-out patients were excluded from inferential statistics tests.

Patients' age, gender, skin phototype, history of skin cancer [both non-melanoma skin cancer (NMSC) and skin melanoma] and number of AKs in the test area are described in Table 1. A statistical difference was seen only for gender distribution $(P=0.005)$.

Patients with a clearance of all lesion (100\% CR) were 6 (23.07\%) with MAL-PDT, 9 (30\%) with IMB and $4(14.28 \%)$ with DHA $(P=0.359)$.

All clinical variables (number and cumulative AKs' area) showed a statistically significant reduction with all treatments (Table 2).

Kruskal-Wallis test for multiple independent samples showed a statistically significant difference of number and total AKs area in the selected test area $(\Delta=\mathrm{T} 0-\mathrm{T} 1)$ with respect to treatment choice $(P<0.001$; Table 3, Fig. 2).

Mann-Whitney test was then used to analyse two by two which treatment was more effective. The test showed a significantly greater reduction in AKs number with PDT in comparison to DHA. We also found that, although the reduction in AKs number with PDT was greater in comparison to IMB and with IMB in comparison to DHA, the differences were not statistically significative. The cumulative AKs area reduced significantly

Table 1 Patients' features of interest for the present study

\begin{tabular}{|c|c|c|c|c|c|}
\hline & Total $N(\%)$ & PDT $N(\%)$ & IMB $N(\%)$ & DHA $N(\%)$ & $\boldsymbol{P}$ \\
\hline Patients & $84(93.3)$ & $26(28.9)$ & $30(33.3)$ & $28(31.1)$ & \\
\hline Age [median (range)] (years) & $78(49-91)$ & $80(71-89)$ & $76(49-89)$ & $77(54-91)$ & 0.079 \\
\hline \multicolumn{6}{|l|}{ Gender: } \\
\hline Males (\%) & $76(90.5)$ & $26(100)$ & $23(76.7)$ & $27(96.4)$ & 0.005 \\
\hline Females (\%) & $8(9.5)$ & $0(0)$ & $7(23.3)$ & $1(3.6)$ & \\
\hline \multicolumn{6}{|l|}{ Skin Photoype: } \\
\hline I & $2(2.4)$ & $1(3.8)$ & $0(0)$ & $1(5.1)$ & 0.501 \\
\hline ॥ & $39(46.4)$ & $12(46.1)$ & $17(56.7)$ & $10(35.7)$ & \\
\hline III & $43(51.2)$ & $13(50.1)$ & $13(43.3)$ & $17(59.2)$ & \\
\hline IV & $0(0)$ & $0(0)$ & $0(0)$ & $0(0)$ & \\
\hline History of previous NMSC and/or skin melanoma & $36(42.9)$ & $12(46.1)$ & $14(46.7)$ & $10(35.7)$ & 0.645 \\
\hline Number of AKs at baseline [median (range)] & $7(5-13)$ & $9(5-12)$ & $7(5-13)$ & $7(5-12)$ & 0.23 \\
\hline
\end{tabular}

Bold values are statistically significant values. 
Table 2 AKs' number and cumulative area at baseline and EOT (median; range) with the three treatment options

\begin{tabular}{|c|c|c|c|c|}
\hline \multirow{2}{*}{$\begin{array}{l}\text { Treatment } \\
\text { MAL-PDT }\end{array}$} & \multicolumn{2}{|c|}{ AKs' number } & \multicolumn{2}{|c|}{ AKs' cumulative area $\left(\mathrm{cm}^{2}\right)$} \\
\hline & Baseline & 90 days EOT & Baseline & 90 days EOT \\
\hline & $9(5-12)$ & $2(0-4)$ & $4.61(2.06-9.7)$ & $0.47(0-2.1)$ \\
\hline & \multicolumn{2}{|c|}{$P<0.0001$} & \multicolumn{2}{|c|}{$P<0.0001$} \\
\hline \multirow[t]{3}{*}{ IMB } & Baseline & 90 days EOT & Baseline & 90 days EOT \\
\hline & $7(5-13)$ & $2(0-6)$ & $3.62(1.75-5.91)$ & $0.76(0-2.69)$ \\
\hline & \multicolumn{2}{|c|}{$P<0.0001$} & \multicolumn{2}{|c|}{$P<0.0001$} \\
\hline \multirow[t]{3}{*}{ DHA } & Baseline & 90 days EOT & Baseline & 90 days EOT \\
\hline & $7(5-12)$ & $3(0-8)$ & $3.4(1.4-8.1)$ & $1.05(0-7.22)$ \\
\hline & \multicolumn{2}{|c|}{$P<0.0001$} & \multicolumn{2}{|c|}{$P<0.0001$} \\
\hline
\end{tabular}

Bold values are statistically significant values.

Table 3 Comparison of AKs' number and cumulative area $\left(\mathrm{cm}^{2}\right)$ in the $25 \mathrm{~cm}^{2}$ field according to treatment options $(\Delta=\mathrm{T} 0-\mathrm{T} 1)$

\begin{tabular}{lll} 
Treatment $^{*}$ & AKs' number & AKs' cumulative area \\
& $P<0.0001$ & $P<0.0001$ \\
\hline PDT vs. IMB $\dagger$ & $P=0.08$ & $P=0.006$ \\
& $\Delta=7$ vs. $\Delta=5.5$ & $\Delta=3.38$ vs. $\Delta=2.81$ \\
PDT vs. DHA $\dagger$ & $P<0.0001$ & $P<0.0001$ \\
& $\Delta=7$ vs. $\Delta=4$ & $\Delta=3.38$ vs. $\Delta=2.34$ \\
IMB vs. DHA $\dagger$ & $P=0.06$ & $P=0.035$ \\
& $\Delta=5.5$ vs. $\Delta=4$ & $\Delta=2.81$ vs. $\Delta=2.34$ \\
\hline
\end{tabular}

${ }^{*}$ Kruskal-Wallis test.

†Mann-Whitney test.

Bold values are statistically significant values.

more with PDT compared to other treatment options and with IMB in comparison to DHA (Table 3).

At HFUS evaluation, total, epidermal and dermal thicknesses of the target AKs changed with all treatments but a statistically significative difference was found only for the reduction in the epidermal thickness with MAL-PDT (Table 4). PDT was also the only treatment option that increased at a significant level the dermal density in both the skin underlying the target AK and the surrounding skin and decreased significantly the SLEB thickness in the perilesional skin (Table 4).

The two by two comparison by Mann-Whitney test of the effects ( $\Delta=$ baseline-90 days after EOT) on total, epidermal and dermal thicknesses of the target AK with the three treatments did not demonstrate any statistically significative difference $(P>0.05)$.

Photodynamic therapy induced a significantly more substantial reduction in SLEB thickness as compared to both IMB and DHA and also an increase in echogenicity of the dermis underlying the target $\mathrm{AK}$ and the surrounding skin as compared to IMB. There was no statistically significative difference between changes of lesional and perilesional dermal echogenicity with DHA and PDT. DHA showed higher $\Delta$ values than IMB of lesional and perilesional dermal echogenicities and SLEB thickness as compared to IMB without reaching a statistical significance (Table 5).
The regression performed to identify whether HFUS baseline dermal density, SLEB thickness and epidermal thickness had a predictive value of therapeutic response did not show statistically significant results ( $>0.05$ for all).

Patients' age and skin phototype were never found predictive of clinical results and variation in HFUS features with all treatment options $(P>0.05)$.

\section{Discussion}

In the present randomized non-inferiority trial, all investigated treatment options were found effective for the treatment of patients with multiple Olsen II AKs. Results show some difference from previously published results (as summarized in ${ }^{15}$ ) but we emphasize that, unlike previous investigations, we assessed the clinical result not only visually but also with digital dermoscopy that allows a better diagnostic accuracy. ${ }^{3,4}$ Another variable that can explain differences in results between studies is the different ratio of $\mathrm{AK} \mathrm{I}$, which are more responsive to all treatments than AK II. ${ }^{22-24}$ However, in the present trial, we enrolled only patients with Olsen II AKs in the target area.

The two by two comparison of treatments has given efficacy results that were different according to the three performance indexes that we used: the rate of patients attaining the clearance of all lesions, the rate of cleared lesions and the reduction in the cumulative AK area.

The rate of patients who achieved the clearance of all lesions was not different with the three treatments. However, this performance index can clearly be heavily influenced by the number of AKs per patient and indeed, in the present trial, it was higher in patients treated with PDT.

These results of the comparison of IMB versus DHA are in contrast with those of a previous study in which IMB was found significantly more effective $(P<0.001)$ than DHA (twice daily for 90 days): $53 \%$ of patients with complete AK clearance (AKCLEAR 100) vs. 45\%, respectively. ${ }^{23}$ However, a different IMB treatment protocol was used as a second treatment cycle with IMB was allowed if the first was no or partially effective. ${ }^{23}$ In another study with a prolonged follow-up (12 months after EOT), the cumulative probability of remaining free from treatment failure (patients attaining $<75 \%$ CR of lesions) was significantly higher with MAL-PDT (37.7\%; 95\% CI: 30.0-45.3) in comparison to IMB (28.9\%; 95\% CI: $21.8-36.3){ }^{24}$

If we look at the second performance index, the rate of cleared AKs in the treated area, PDT was found superior to DHA whereas the comparisons of PDT versus IMB and IMB versus DHA did not show statistically significant differences.

This performance index is often considered the most reliable, ${ }^{14,17}$ although it can be influenced by the size of lesions and by the difficulty of calculating the AKs number when lesions are close each other or partially confluent.

Nonetheless, the results of the present study substantially confirm the results of two previous randomized comparative trials 

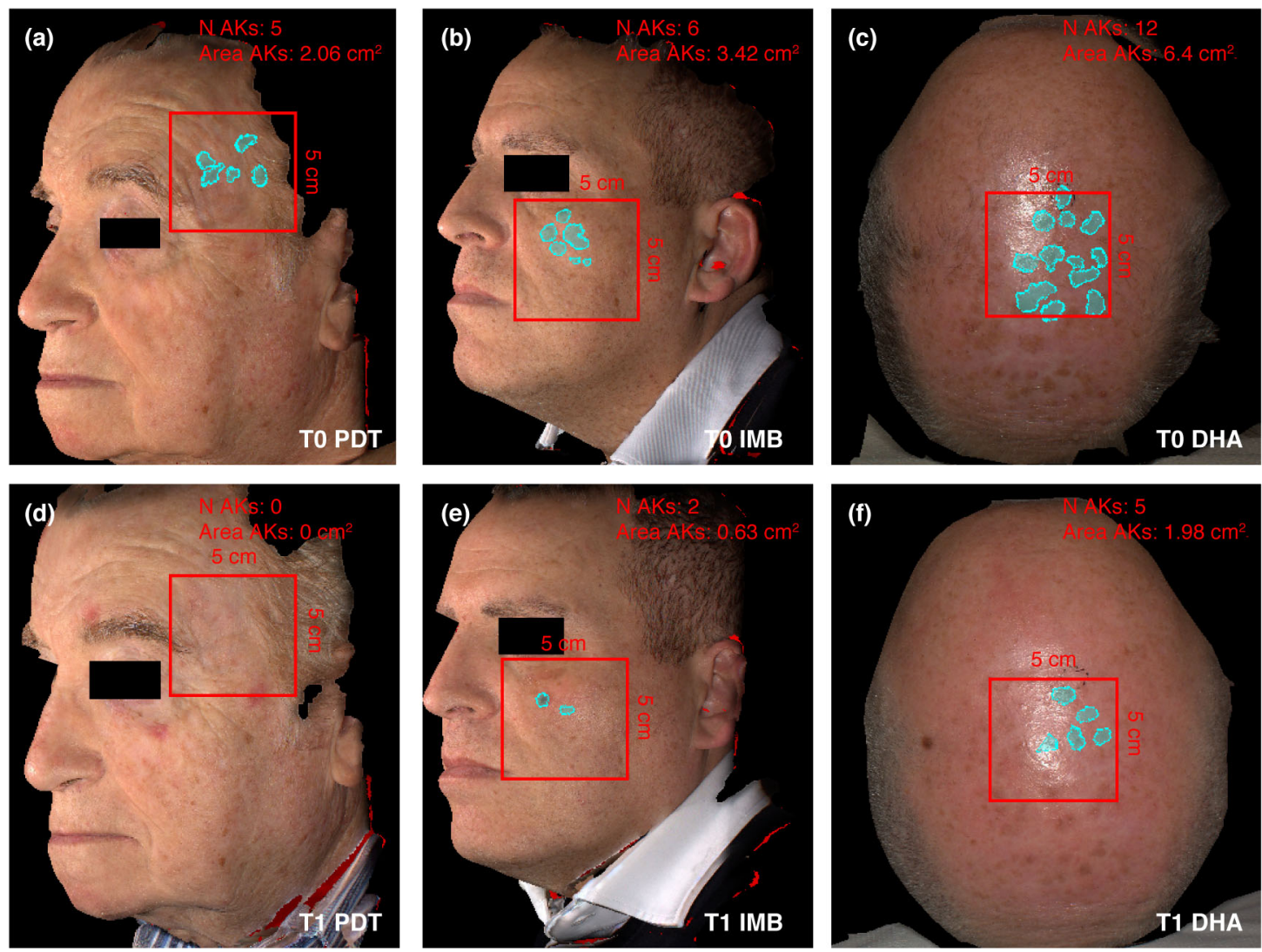

Figure 2 Clinical evaluation of AKs' number and cumulative area (turquoise area, $\mathrm{cm}^{2}$ ) in the $25 \mathrm{~cm}^{2}$ field by 3D digital pictures (a. PDT baseline; b. IMB baseline; c. DHA baseline; d. PDT 90 days from EOT; e. IMB 90 days from EOT; f. DHA 90 days from EOT). AK, actinic keratosis; DHA, diclofenac plus hyaluronate gel; IMB, ingenol mebutate gel; PDT, photodynamic therapy.

from our centre. In the first study, MAL-PDT was found more effective than IMB (AKs' clearance rates $42.9 \%$ and $31.4 \%$, respectively) but the difference did not reach a statistical significativity $(P=\mathrm{NS}) .{ }^{25}$ In the second study, the lesion CR rates at 3 months from the EOT were $85.9 \%$ and $51.8 \%(P<0.0001)$ and the patient CR rates were $68 \%$ and $27 \%(P<0.0001)$ with MAL-PDT (a treatment cycle followed by a second, if needed after 3 months) and DHA (twice daily for 90 days), respectively. ${ }^{26}$ MAL-PDT was significantly superior also for cosmetic outcome and patient's overall satisfaction. ${ }^{26}$

Table 4 Significant HFUS parameters (median; range) at baseline (TO) and 90 days after EOT (T1)

\begin{tabular}{|c|c|c|c|c|c|c|c|}
\hline Treatment & & $\begin{array}{l}\text { Target AK total } \\
\text { thickness } \\
(\mu \mathrm{m})\end{array}$ & $\begin{array}{l}\text { Target AK } \\
\text { epidermal } \\
\text { thickness }(\mu \mathrm{m})\end{array}$ & $\begin{array}{l}\text { Target AK dermal } \\
\text { thickness } \\
(\mu \mathrm{m})\end{array}$ & $\begin{array}{l}\text { Target AK dermal } \\
\text { density } \\
(\%)\end{array}$ & $\begin{array}{l}\text { Perilesional } \\
\text { dermal density } \\
\text { (\%) }\end{array}$ & $\begin{array}{l}\text { Perilesional SLEB } \\
\text { thickness } \\
(\mu \mathrm{m})\end{array}$ \\
\hline \multirow[t]{3}{*}{ MAL-PDT } & TO & 1176 (797-1836) & $261(39-531)$ & 984.5 (695-1297) & $11.85(2.04-34.81)$ & $10.92(3.72-51.08)$ & $152(102-500)$ \\
\hline & $\mathrm{T} 1$ & 1117.5 (742-1609) & $171.5(54-672)$ & $832(86-1406)$ & $14.82(3.2-29.14)$ & $21.15(2.5-74)$ & $113(31-234)$ \\
\hline & $P$ & 0.082 & 0.04 & 0.101 & 0.03 & 0.008 & $<0.001$ \\
\hline \multirow[t]{3}{*}{$\mathrm{DHA}$} & TO & 1008 (742-1813) & $140(85-586)$ & $867(641-1406)$ & $9.3(3.63-27.18)$ & $9.8(3.04-34.62)$ & $168(78-453)$ \\
\hline & $\mathrm{T} 1$ & $1144.5(750-1750)$ & $154(63-655)$ & 957 (555-1484) & $9.26(3.8-43.66)$ & $8.32(4.22-41.64)$ & $168(76-398)$ \\
\hline & $P$ & 0.355 & 0.991 & 0.226 & 0.586 & 0.805 & 0.229 \\
\hline \multirow[t]{3}{*}{ IMB } & TO & 1007 (703-1648) & $110.5(47-656)$ & 773.5 (586-1320) & $10.91(1.85-29.33)$ & $10.16(4.54-35.02)$ & $148(78-258)$ \\
\hline & $\mathrm{T} 1$ & 1008 (729-1836) & $117.5(41-453)$ & 793 (644-1226) & $12.82(1.66-40.29)$ & $10.39(5.39-52.04)$ & $136(55-484)$ \\
\hline & $P$ & 0.345 & 0.838 & 0.657 & 0.122 & 0.88 & 0.71 \\
\hline
\end{tabular}

Bold values are statistically significant values. 


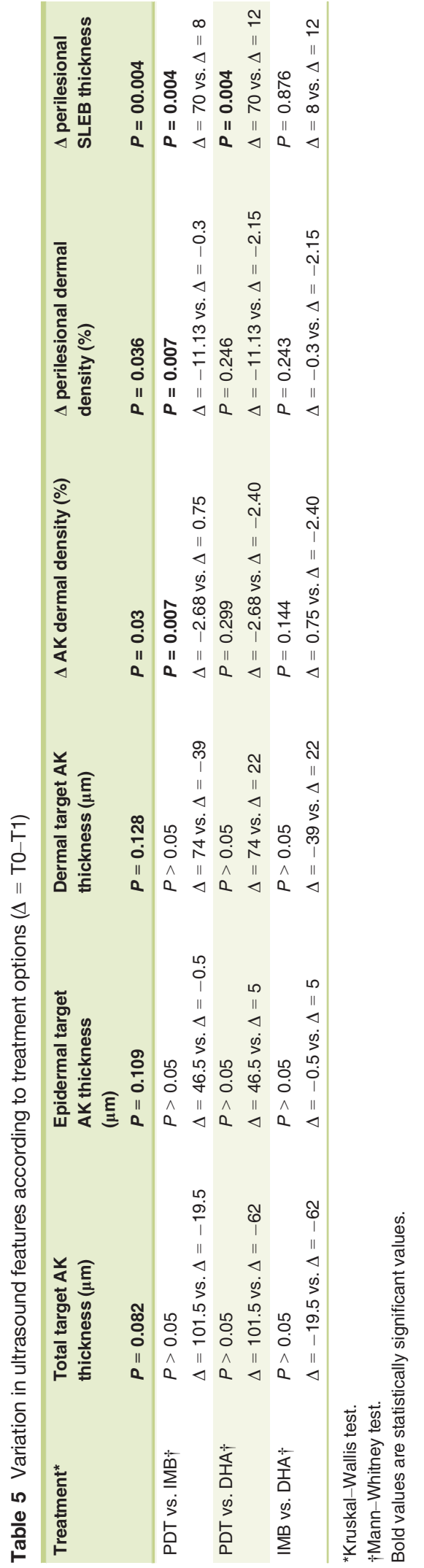

Because of the limitations of the first two performance indexes, we have proposed a new morphometric performance index that is the reduction in the cumulative AKs area in the treated field. It is objective because it is calculated digitally from $3 \mathrm{D}$ pictures using a dedicated analysis software, regardless of the number of individual lesions. This parameter improved at a statistically significative level, with PDT in comparison to both DHA and IMB and for IMB in comparison to DHA. These results support the usefulness of image-guided prediction of treatment efficacy in dermatology.

In the present trial, we also aimed to investigate and to compare the effect of the three treatments on the HFUS parameters of both lesional and surrounding chronically photo-damaged skin.

The thickness of the epidermal layer of the AK spots after all treatments was similar. In the groups treated with IMB and DHA, it was similar to baseline whereas the thickness significantly decreased with MAL-PDT. However, we must take into account that the average baseline epidermal thickness of AK spots was higher in this group. The total and dermal thickness of AK spots did not change with all treatments suggesting that the clearance of lesions is not followed by skin atrophy.

The two main HFUS features of photodamage are the dermal echogenicity, that correlates to its density, and the thickness of the subepidermal low-echogenic band (SLEB). ${ }^{27}$

MAL-PDT was the only treatment option that increased significantly the dermal density underlying and surrounding the AK spots and decreased significantly the SLEB density of the perilesional skin (Fig. 3).

The two by two comparison of drug results shows that all these echographic dermal effects were significantly more
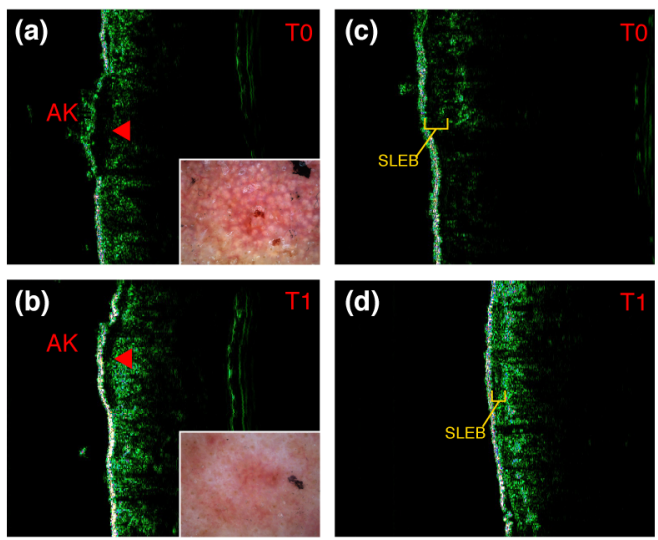

Figure 3 HFUS features of a target AK (red arrow) before and after MAL-PDT $(a, b)$ and its dermoscopy (detail). SLEB thickness and density of the surrounding photo-damaged area before and after treatment (c,d). HFUS, high-frequency ultrasound; AK, actinic keratosis; MAL-PDT, MAL-photodynamic therapy; SLEB, subepidermal low-echogenic band. 
pronounced with MAL-PDT in comparison to IMB, whereas only the reduction in SLEB, but not dermal density, was more pronounced with MAL-PDT in comparison to DHA whereas no difference was found between IMB and DHA for all HFUS features.

The increase in the echogenicity of the upper and lower dermal layers and the reduction in the SLEB thickness with MALPDT can be related to several concurrent biological effects: the reduction in the neo-angiogenic microvascularization, ${ }^{28-30}$ the reduction in the dermal inflammatory infiltrate, ${ }^{31}$ the fibroblasts proliferation and collagen synthesis ${ }^{32,33}$ and the removal of elastotic fibres by macrophages. ${ }^{32,33}$

Altogether these biological activities can explain the photorejuvenative effect of MAL-PDT that was previously demonstrated clinically. ${ }^{6,31}$ Theoretically, the improvement of dermal photodamage could also reduce the pathogenetic role of damaged dermis in the development of AKs. ${ }^{34}$

In the present investigation, the increase of the echogenicity of the upper and lower dermal layers and perilesional SLEB was detected with DHA treatment as well, and it could be mainly related to effects on COX-2 inhibition and consequent downregulation of E2 prostaglandin (PGE2) and angiogenesis. ${ }^{35}$

However, a clinical photorejuvenative effect with DHA has not been reported so far.

In the present investigation, IMB had no effect on HFUS parameters of dermal photodamage. However, a rejuvenation effect on clinical features of photodamage has been recently described in a preliminary study, ${ }^{36}$ and therefore, further studies are needed to clarify this issue.

Another aim of the present study was to identify possible predictors of therapeutic response of the three therapies. However, either baseline clinical parameters (age and skin phototype) or baseline HFUS parameters (baseline epidermal thickness, dermal density and SLEB thickness) were not found to be predictive of the therapeutic response with each treatment. In this field, HFUS may not be the most appropriate tool and other non-invasive techniques, such as reflectance confocal microscopy, may add some more information.

In conclusion, the present investigation has demonstrated that MAL-PDT is more effective than IMB and DHA for reducing the cumulative AK area, more effective than DHA, but not IMB, for reducing the AK number whereas no difference was seen in the number of patients with all cleared lesions. In our opinion, the digital assessment of the reduction in the cumulative AK area (preceded by a careful inspection of the treated area with digital dermoscopy) should be the preferred performance index for the evaluation of the efficacy of treatments for AKs. In addition, we have demonstrated that all AK treatments restore the epidermal thickness and that all treatments are not followed by skin atrophy. However, only MAL-PDT improves all HFUS features of chronic photodamages of the dermis of the skin underlying and surrounding the AK spots.

\section{References}

1 Dréno B, Amici JM, Basset-Seguin N, Cribier B, Claudel JP, Richard MA, AKTeam $^{\mathrm{TM}}$. Management of actinic keratosis: a practical report and treatment algorithm from AKTeam ${ }^{\mathrm{TM}}$ expert clinicians. J Eur Acad Dermatol Venereol 2014; 28: 1141-1149.

2 Olsen EA, Abernethy ML, Kulp-Shorten C et al. A double-blind, vehicle controlled study evaluating masoprocol cream in the treatment of actinic keratoses on the head and neck. J Am Acad Dermatol 1991; 24: 738-743.

3 Zalaudek I, Giacomel J, Schmid K et al. Dermatoscopy of facial actinic keratosis, intraepidermal carcinoma, and invasive squamous cell carcinoma: a progression model. J Am Acad Dermatol 2012; 66: 589-597.

4 Guitera P, Menzies SW, Argenziano G et al. Dermoscopy and in vivo confocal microscopy are complementary techniques for diagnosis of difficult amelanotic and light-coloured skin lesions. Br J Dermatol 2016; 175: 1311-1319.

5 Mogensen M, Nürnberg BM, Forman JL, Thomsen JB, Thrane L, Jemec GB. In vivo thickness measurement of basal cell carcinoma and actinic keratosis with optical coherence tomography and 20-MHz ultrasound. $\mathrm{Br}$ J Dermatol 2009; 160: 1026-1033.

6 Zane C, Capezzera R, Sala R, Venturini M, Calzavara-Pinton P. Clinical and echographic analysis of photodynamic therapy using methylaminolevulinate as sensitizer in the treatment of photodamaged facial skin. Lasers Surg Med 2007; 39: 203-209.

7 Bobyr I, Campanati A, Consales V et al. Ingenol mebutate in actinic keratosis: a clinical, videodermoscopic and immunohistochemical study. J Eur Acad Dermatol Venereol 2017; 31: 260-266.

8 Peris K, Calzavara-Pinton PG, Neri L et al. Italian expert consensus for the management of actinic keratosis in immunocompetent patients. J Eur Acad Dermatol Venereol 2016; 30: 1077-1084.

9 Fernández-Figueras MT, Carrato C, Sáenz X et al. Actinic keratosis with atypical basal cells (AK I) is the most common lesion associated with invasive squamous cell carcinoma of the skin. J Eur Acad Dermatol Venereol 2015; 29: 991-997.

10 Dirschka T, Pellacani G, Micali G et al. A proposed scoring system for assessing the severity of actinic keratosis on the head: actinic keratosis area and severity index. J Eur Acad Dermatol Venereol 2017; 31: 12951302 .

11 de Berker D, McGregor JM, Mohd Mustapa MF, Exton LS, Hughes BR. British Association of Dermatologists' guidelines for the care of patients with actinic keratosis 2017. Br J Dermatol 2017; 176: 20-43.

12 Morton CA. A synthesis of the world's guidelines on photodynamic therapy for non-melanoma skin cancer. G Ital Dermatol Venereol 2018; 153: 783-792.

13 Werner RN, Stockfleth E, Connolly SM et al. Evidence- and consensusbased (S3) Guidelines for the Treatment of Actinic Keratosis - International League of Dermatological Societies in cooperation with the European Dermatology Forum - Short version. J Eur Acad Dermatol Venereol 2015; 29: 2069-2079.

14 Dirschka T, Gupta G, Micali G et al. Real-world approach to actinic keratosis management: practical treatment algorithm for office-based dermatology. J Dermatolog Treat 2017; 28: 431-442.

15 Vegter S, Tolley K. A network meta-analysis of the relative efficacy of treatments for actinic keratosis of the face or scalp in Europe. PLoS ONE 2014; 9: e96829.

16 Calzavara-Pinton P, Zane C, Pacou M, Szeimies RM. Bucher's indirect comparison of daylight photodynamic therapy with methyl aminolevulinate cream versus diclofenac plus hyaluronic acid gel for the treatment of multiple actinic keratosis. Eur J Dermatol 2016; 26: 487-492.

17 Calzavara-Pinton P, Hædersdal M, Barber K et al. Structured expert consensus on actinic keratosis: treatment algorithm focusing on daylight PDT. J Cutan Med Surg 2017. https://doi.org/10.1177/1203475417702994 [Epub ahead of print].

18 Calzavara-Pinton P, Zane C, Arisi M, Hamon PA, Tanova NT. Evaluation of the costs of topical treatments for actinic keratosis based on lesion 
response and the affected area. G Ital Dermatol Venereol 2018; 153: 764775 .

19 URL https://www.drugs.com/picato.html (last accessed 07 July 2019)

20 URL https://www.drugs.com/uk/diclofenac-sodium-3-gel-leaflet.html (last accessed 07 July 2019

21 URL https://www.drugs.com/metvixia.html (last accessed 07 July 2019)

22 Szeimies RM, Karrer S, Radakovic-Fijan S et al. Photodynamic therapy using topical methyl 5-aminolevulinate compared with cryotherapy for actinic keratosis: a prospective, randomized study. J Am Acad Dermatol 2002; 47: 258-262.

23 Stockfleth E, Harwood CA, Serra-Guillén C, Larsson T, Østerdal ML, Skov T. Phase IV head-to-head randomized controlled trial comparing ingenol mebutate $0015 \%$ gel with diclofenac sodium $3 \%$ gel for the treatment of actinic keratosis on the face or scalp. Br J Dermatol 2018; 178: 433-442.

24 Jansen MHE, Kessels JPHM, Nelemans PJ et al. Randomized trial of four treatment approaches for actinic keratosis. N Engl J Med 2019; 380: 935946.

25 Zane C, Fabiano A, Arisi M, Calzavara-Pinton P. A randomized split-face clinical trial of photodynamic therapy with methyl aminolevulinate versus ingenol mebutate gel for the treatment of multiple actinic keratoses of the face and scalp. Dermatology 2016; 232: 472-477.

26 Zane C, Facchinetti E, Rossi MT, Specchia C, Calzavara-Pinton PG. A randomized clinical trial of photodynamic therapy with methyl aminolaevulinate vs. diclofenac 3\% plus hyaluronic acid gel for the treatment of multiple actinic keratoses of the face and scalp. Br J Dermatol 2014; 170: 1143-1150.

27 Sandby-Møller J, Wulf HC. Ultrasonographic subepidermal low-echogenic band, dependence of age and body site. Skin Res Technol 2004; 10: 57-63.
28 Middelburg TA, de Bruijn HS, Tettero L et al. Topical hexylaminolevulinate and aminolevulinic acid photodynamic therapy: complete arteriole vasoconstriction occurs frequently and depends on protoporphyrin IX concentration in vessel wall. J Photochem Photobiol, B 2013; 126: 26-32.

29 Becker TL, Paquette AD, Keymel KR, Henderson BW, Sunar U. Monitoring blood flow responses during topical ALA-PDT. Biomed Opt Express 2010; 2: 123-130.

30 Middelburg TA, Kanick SC, de Haas ER et al. Monitoring blood volume and saturation using superficial fibre optic reflectance spectroscopy during PDT of actinic keratosis. J Biophotonics 2011; 4: 721-730.

31 Szeimies RM, Torezan L, Niwa A et al. Clinical, histopathological and immunohistochemical assessment of human skin field cancerization before and after photodynamic therapy. Br J Dermatol 2012; 167: 150159

32 Orringer JS, Hammerberg C, Hamilton T et al. Molecular effects of photodynamic therapy for photoaging. Arch Dermatol 2008; 144: 1296-1302.

33 Wang P, Han J, Wei M et al. Remodeling of dermal collagen in photoaged skin using low-dose 5-aminolevulinic acid photodynamic therapy occurs via the transforming growth factor- $\beta$ pathway. J Biophotonics 2018; 11: e201700357.

34 Procopio MG, Laszlo C, Al Labban D et al. Combined CSL and p53 downregulation promotes cancer-associated fibroblast activation. Nat Cell Biol 2015; 17: 1193-1204.

35 Zhan $\mathrm{H}$, Zheng $\mathrm{H}$. The role of topical cyclo-oxygenase-2 inhibitors in skin cancer: treatment and prevention. Am J Clin Dermatol 2007; 8: 195-200.

36 Kim M, Jung Y, Kim J, Jeong SW, Woo YR, Park HJ. Anti-aging effects of ingenol mebutate for patients with actinic keratosis. J Am Acad Dermatol 2018; 79: 1148-1150. 